\title{
Associated Anomalies in Congenital Lung Abnormalities: A 20-Year Experience
}

\author{
Sergei M. Hermelijn ${ }^{a}$ Rutger R. Zwartjes ${ }^{b}$ Harm A.W.M. Tiddens ${ }^{b, c}$ \\ Suzan C.M. Cochius-den Otter ${ }^{a}$ Irwin K.M. Reiss ${ }^{d}$ René M.H. Wijnen ${ }^{a}$ \\ J. Marco Schnater ${ }^{\mathrm{a}}$
}

a Department of Paediatric Surgery and Intensive Care, Erasmus University Medical Centre, Sophia Children's Hospital, Rotterdam, The Netherlands; ' Department of Paediatric Pulmonology, Erasmus University Medical Centre, Sophia Children's Hospital, Rotterdam, The Netherlands; ${ }^{C}$ Department of Radiology and Nuclear Medicine, Erasmus University Medical Centre, Rotterdam, The Netherlands; ${ }^{\mathrm{d}}$ Department of Neonatology, Erasmus University Medical Centre, Sophia Children's Hospital, Rotterdam, The Netherlands

\section{Keywords}

Congenital lung disease - Lung malformation · Paediatric pulmonology · Congenital cystic adenomatoid malformation - Congenital pulmonary airway malformation

\section{Abstract}

Introduction: A congenital lung abnormality (CLA) is often found in conjunction with other abnormalities but screening guidelines for newborns with CLA have not yet been reported. We aimed to assess the incidence of associated anomalies in CLA patients born or followed up at our centre and the need for additional screening of newborns with a CLA. Methods: From a retrospective chart review of all patients born with a CLA between January 1999 and January 2019, we identified patients diagnosed with a congenital pulmonary airway malformation, bronchopulmonary sequestration, congenital lobar overinflation, bronchogenic cyst, or lung agenesis. Associated anomalies were noted and categorized according to the affected organ system. Results: Twentyeight (14\%) of 196 CLA patients had a major associated anomaly. This was most frequent in conjunction with a lung agenesis $(100 \%)$ or bronchogenic cyst (29\%). Congenital heart defects (32\%) and gastrointestinal defects (18\%) were the most frequently associated anomalies. Examination of

karger@karger.com www.karger.com/neo

Karger"

GOPEN ACCESS (c) 2020 The Author(s)

Published by S. Karger AG, Basel

This article is licensed under the Creative Commons AttributionNonCommercial-NoDerivatives 4.0 International License (CC BY NC-ND) (http://www.karger.com/Services/OpenAccessLicense). Usage and distribution for commercial purposes as well as any distribution of modified material requires written permission. newborns with a CLA should focus on the cardiovascular and gastrointestinal tract, and a chest and abdominal radiograph may be useful to assess signs of major associated anomalies, regardless of the clinical course.

(c) 2020 The Author(s)

Published by S. Karger AG, Basel

\section{Introduction}

The incidence of a congenital lung abnormality (CLA) has increased to $4 / 10,000$ births after the introduction of routine prenatal ultrasound screening and CLAs are most often found in conjunction with other abnormalities [1, 2]. The most common CLAs, in order of prevalence, are congenital pulmonary airway malformation (CPAM), bronchopulmonary sequestration (BPS), congenital lobar overinflation (CLO, previously known as congenital lobar emphysema), bronchogenic cyst (BC), and lung agenesis.

Most newborns with a CLA are asymptomatic at birth and the majority remain asymptomatic during childhood [3]. The diagnosis is confirmed with a postnatal CT scan [4], after which the lesion is either resected or followed up, depending on the clinical symptoms, local guidelines, and standard of care $[3,5]$. 
Table 1. Patient characteristics

\begin{tabular}{|c|c|c|}
\hline & \multicolumn{2}{|l|}{ Associated anomalies } \\
\hline & no $(n=168 ; 86)$ & yes $(n=28 ; 14)$ \\
\hline Male gender & $93(55)$ & $12(43)$ \\
\hline Gestational age at birth, weeks & $39.1(31.7-42.3)$ & $38.8(30.4-41.4)$ \\
\hline Birth weight, $g$ & $3,258(1,650-4,600)$ & $3,115(1,580-3,880)$ \\
\hline Apgar score after $1 \mathrm{~min}$ & $9(1-10)$ & $8(0-10)$ \\
\hline Apgar score after $5 \mathrm{~min}$ & $9(5-10)$ & $9(4-10)$ \\
\hline \multicolumn{3}{|l|}{ Types of congenital lung abnormality* } \\
\hline CPAM (congenital pulmonary airway malformation) & $91(54)$ & $12(43)$ \\
\hline BPS (bronchopulmonary sequestration) & $37(22)$ & $4(14)$ \\
\hline CLO (congenital lobar overinflation) & $30(18)$ & $3(11)$ \\
\hline BC (bronchogenic cyst) & $10(6)$ & $4(14)$ \\
\hline Lung agenesis & - & $3(11)$ \\
\hline Multiple abnormalities & - & $2(7)$ \\
\hline \multicolumn{3}{|l|}{ Location of abnormality on imaging* } \\
\hline RUL & $14(8)$ & $1(4)$ \\
\hline RML & $3(2)$ & $1(4)$ \\
\hline RLL & $58(35)$ & $9(32)$ \\
\hline LUL & $20(12)$ & $2(7)$ \\
\hline LLL & $49(29)$ & $4(14)$ \\
\hline Multiple & $20(12)$ & $8(29)$ \\
\hline Extrapulmonary & $4(2)$ & $3(11)$ \\
\hline Surgical resection due to symptoms* & $56(34)$ & $15(54)$ \\
\hline Maternal age at full-term date & $31(20-44)$ & $30(23-37)$ \\
\hline Gravidity & $2(1-9)$ & $2(1-12)$ \\
\hline Parity & $1(0-7)$ & $0(0-2)$ \\
\hline \multicolumn{3}{|l|}{ Associated anomalies (EUROCAT categories) } \\
\hline Congenital heart defects & & $9(32)$ \\
\hline Digestive system & & $5(18)$ \\
\hline Multiple congenital anomalies & & $3(11)$ \\
\hline Genetic syndromes & & $3(11)$ \\
\hline Vascular & & $2(7)$ \\
\hline Limb & & $2(7)$ \\
\hline Other respiratory & & $1(4)$ \\
\hline Urinary & & $1(4)$ \\
\hline Genital & & $1(4)$ \\
\hline Other anomalies & & $1(4)$ \\
\hline
\end{tabular}

Data are presented as $n(\%)$ or median (range). ${ }^{*} p<0.05$. RUL, right upper lobe; RML, right middle lobe; RLL, right lower lobe; LUL, left upper lobe; LLL, left lower lobe.

It is hypothesized that a CLA arises during the embryonic development of the lungs, either due to interruption of the normal organogenesis or a mismatch of the epithelial-mesenchymal interaction caused by dysregulation of cellular signaling [6]. The type of CLA is thought to be dependent on the timing of the dysregulation $[6,7]$. This dysregulation may render other organ systems susceptible to developmental defects as well [8]. To the best of our knowledge, the incidence of associated anomalies in newborns with a CLA have not yet been reported and screening guidelines are lacking. We aimed to assess the inci- dence of associated anomalies in CLA patients born and followed up at our centre and the need for additional screening of newborns with a CLA.

\section{Materials and Methods}

We retrospectively collected information from the electronic patient records of all CLA patients referred between January 1999 and January 2019 to the Erasmus Medical Centre, Sophia Children's Hospital, Rotterdam, The Netherlands, which is a level III perinatal referral centre. Patients included in this study 


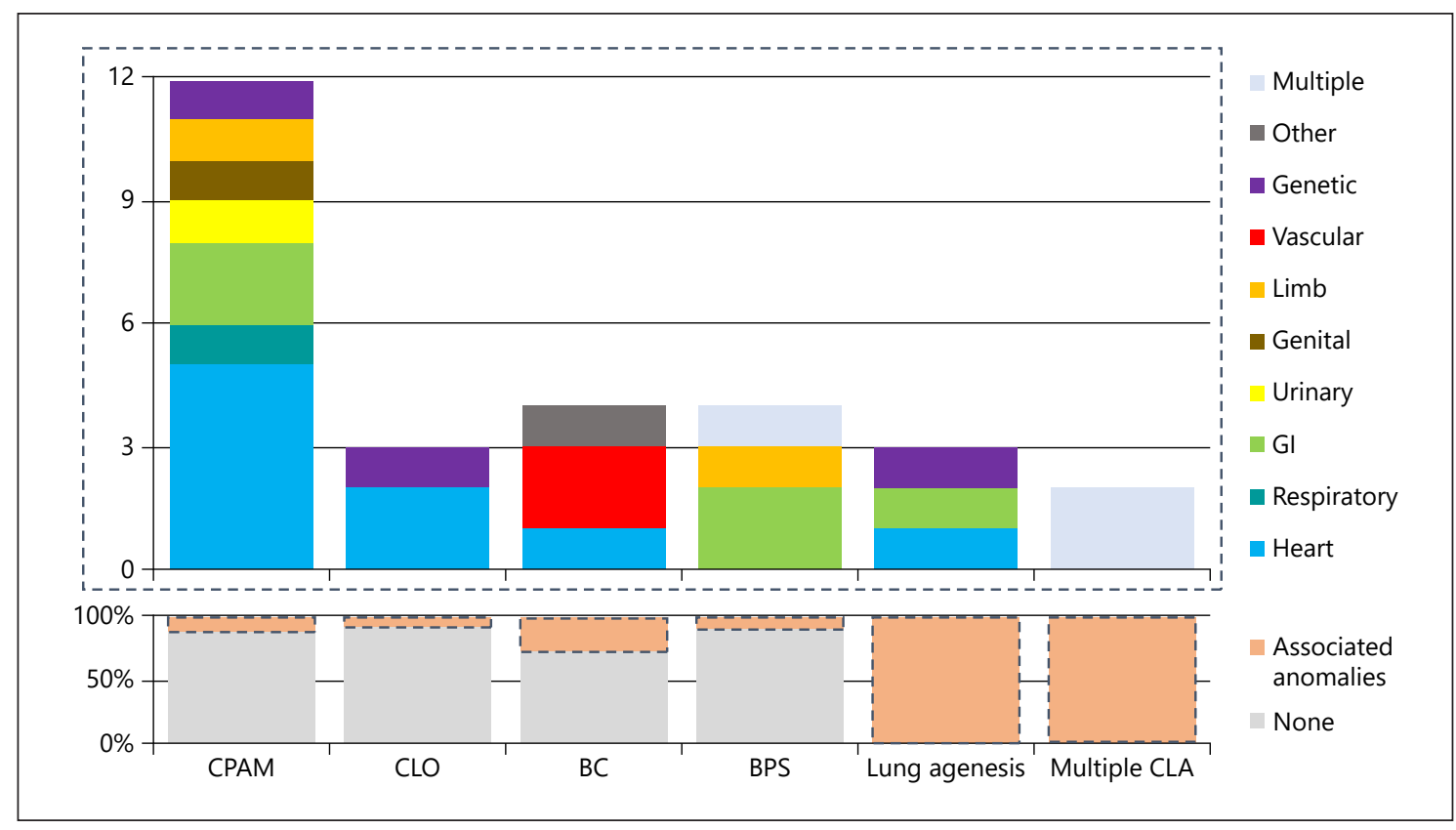

Fig. 1. Bottom bar chart shows the proportion of associated anomalies (orange) for each congenital lung abnormality. Top bar chart (within dashed line) specifies the number of patients with associated anomalies and the affected organ systems. CPAM, congenital pulmonary airway malformation; BPS, bronchopulmonary sequestration; CLO, congenital lobar emphysema; BC, bronchogenic cyst; GI, gastrointestinal tract.

were those with a postnatal diagnosis of CPAM, BPS, CLO, BC, or lung agenesis. CPAM was defined as either a dominant cyst or a conglomerate of multiple cysts without systemic arterial blood supply, and the latter is the hallmark of a BPS. BC was defined as a solitary cystic structure with a close relation to the mediastinum, and CLO was defined as a hyperinflated lung lobe. Hybrid BPS, defined as solid lung tissue with adjacent cysts receiving systemic arterial blood supply, was regarded as a single abnormality. These abnormalities were diagnosed on the basis of either a postnatal chest CT scan or histological examination in cases of surgical resection. At our centre, a postnatal chest radiograph is made in the first $24 \mathrm{~h}$, to assess the size and aspect of the lesion if it is visible on a radiograph. Asymptomatic neonates are discharged after $24 \mathrm{~h}$ if no other anomalies are found during the structured newborn examination [9]. We adhere to a waitand-see policy for all CLA patients, unless they exhibit symptoms such as respiratory distress, recurrent infections, persistent cough, or cardiac volume overload, in which case the lesion is surgically resected. All CLA patients are enrolled in our surgical long-term follow-up program which entails structured hospital visits at set ages, during which physical growth, developmental, and lung function tests are performed. We collected information on the child's sex, gestational age at birth, birth weight, Apgar score, diagnosis, location of the abnormality, and history of symptoms. We also collected maternal age at full-term date, gravidity, and parity. All correspondence, notes, and radiology reports were reviewed for associated anomalies which were categorized according to the affected organ system, consistent with the guidelines of the European Registry of Congenital Anomalies (EUROCAT) [10].

Associated Anomalies in Congenital Lung Abnormalities

\section{Statistical Analysis}

Statistical analysis was performed using SPSS v25 (IBM Corp., Armonk, NY, USA) and RStudio v1.0.153 (RStudio, Inc., Boston, MA, USA). We used the "glmnet" and "Ridge" packages for our analyses. Differences between groups with and without associated anomalies were assessed using the Mann-Whitney U test for continuous variables and the $\chi^{2}$ test for categorical variables. To correct for multi-collinearity and overfitting, a logistic ridge regression was performed to predict the presence of associated anomalies. Ridge regression is a form of penalized regression in which coefficients with unimportant terms are driven towards zero. The penalization or ridge parameter was chosen using 10-fold crossvalidation, and we used the lambda with the minimum mean cross-validated error. Coefficients are thus slightly biased downwards but have smaller standard errors (SEs), and are therefore more precise [11]. The 2-tailed statistical significance was set at $p<0.05$ unless the Bonferroni correction for multiple testing was applied.

\section{Results}

In the 20-year period studied, a CLA had been diagnosed in 196 patients, 155 of whom (79\%) were born after the introduction of The Netherlands structured ultrasound programme in 2007. Associated anomalies were found in 28 (14\%) patients, 5 of whom (18\%) were born before 2007. In most cases (82\%), the CLA had been diagnosed prena- 


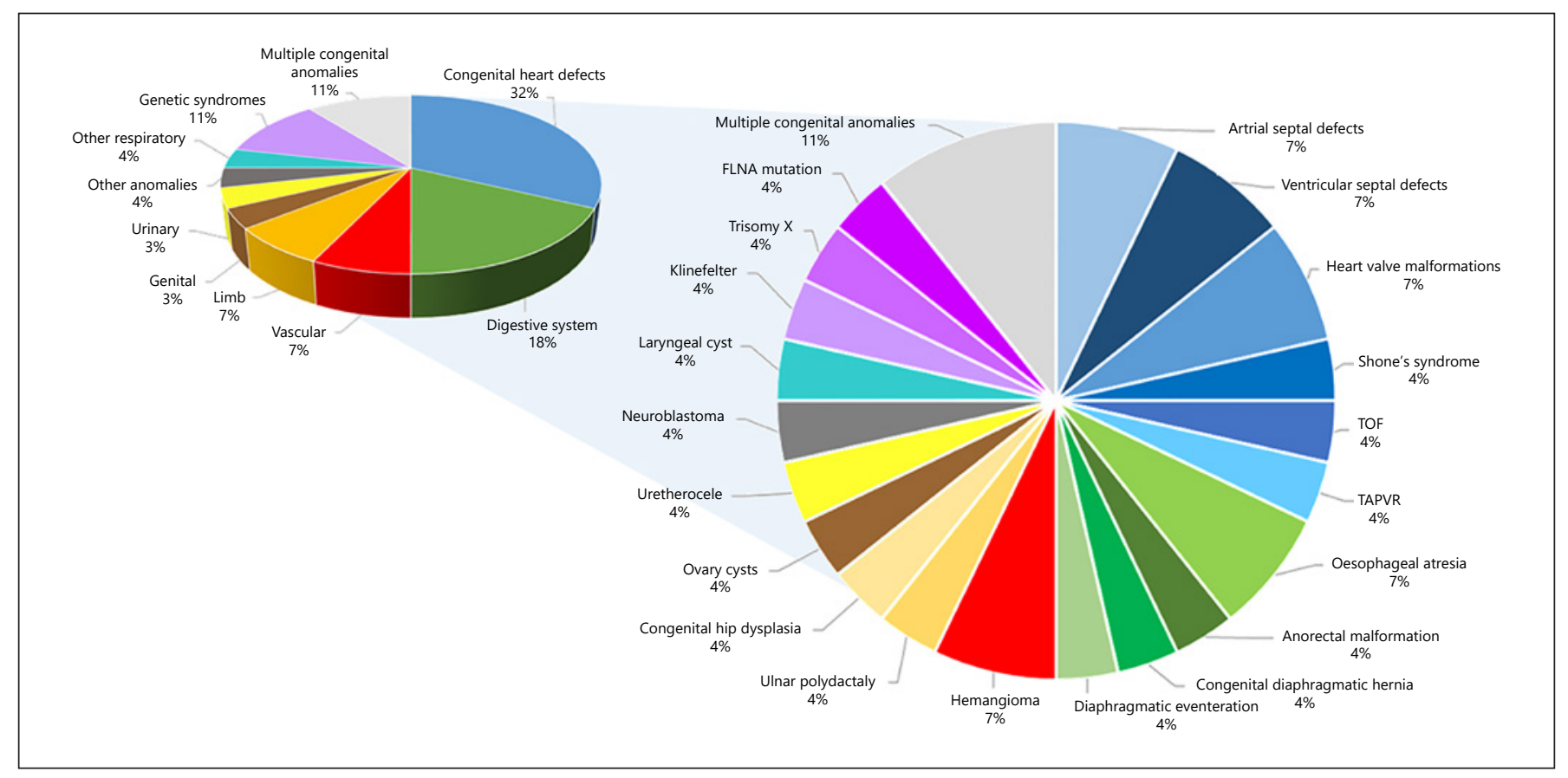

Fig. 2. Distribution of associated anomalies in each anatomical tract (left) and specific anomalies in each tract (right). TAPVR, total anomalous pulmonary venous return; TOF, tetralogy of Fallot.

tally. Most patients were born at term with a median birth weight of 3,240 $\mathrm{g}$ (range 1,580-4,600 $\mathrm{g}$ ) and a median Apgar score of 9 (range 4-10) after 5 min (Table 1). In most cases, a single lung lobe was affected, most commonly a lower lung lobe. In 7 cases, the CLA was not located in the lung parenchyma; 2 were extrapulmonary BPS and 5 were mediastinal BC. Seventy-one (36\%) of all 196 patients underwent a surgical resection of the CLA due to symptoms, and $15(21 \%)$ of these had associated anomalies. Of the 125 asymptomatic patients, 13 (10\%) had associated anomalies. The CLA with the highest proportion of associated anomalies was lung agenesis $(3 / 3 ; 100 \%)$, followed by BC (4/14; 29\%), CPAM (12/103; $12 \%)$, BPS $(4 / 41 ; 10 \%)$, and CLO $(3 / 33 ; 9 \%)$. Two patients had a CLA in each lung. The various associated anomalies were distributed across different CLA types (Fig. 1). Signs of associated anomalies were seen on a chest radiograph in $9(32 \%)$ patients.

Of the 28 patients with associated anomalies, $13(46 \%)$ had been prenatally diagnosed with the anomaly and 11 (39\%) had been diagnosed at the newborn examination. In $4(14 \%)$ patients, the associated anomalies were diagnosed later: 1 with an adrenal neuroblastoma found incidentally, 1 with hip dysplasia diagnosed due to the family history, 1 with a bicuspid aortic valve, and 1 with an atrial septal defect.
Three (11\%) patients with associated anomalies had a genetic syndrome: Klinefelter syndrome, trisomy $\mathrm{X}$, and FLNA gene mutation, respectively (Fig. 2). Three (11\%) other patients had multiple congenital abnormalities. One patient with BPS also had VACTERL association including an anorectal malformation, malrotation of the bowels, bladder exstrophy, duplex kidney, epispadia, persistent ductus arteriosus, and atrial and ventricular septal defects. Another patient had both a BPS and a CPAM. And lastly, 1 patient with lung agenesis also had a BPS and an atrial septal defect.

Excluding genetic and multiple abnormalities, a congenital heart defect was the most common associated anomaly and found in 9 (32\%) patients, 5 of whom needed cardiac surgery and 4 who were routinely monitored. Gastrointestinal defects were found in 5 (18\%) patients; all of the defects were surgically corrected (Fig. 2).

The results of the logistic ridge regression to identify predictors of associated anomalies are summarized in Table 2. Significant predictors of associated anomalies included the diagnosis of $\mathrm{BC}$, lung agenesis, multiple CLA, and an extrapulmonary location of the CLA. The odds of having an associated anomaly are increased by 1.6 for BC, and by 2.3 for extrapulmonary location, lung agenesis, and multiple CLA. 
Table 2. Ridge regression parameters

\begin{tabular}{lrl}
\hline & Coefficient & $p$ value \\
\hline Intercept & 1.88 & n.a. \\
Female & 0.08 & 0.23 \\
Gestational age at birth & 0.01 & 0.69 \\
Birth weight & 0.00 & 0.21 \\
CPAM & -0.02 & 0.72 \\
CLO & -0.08 & 0.40 \\
BC & 0.45 & 0.01 \\
BPS & -0.11 & 0.11 \\
Lung agenesis & 0.85 & 0.04 \\
Multiple CLA & 0.83 & 0.05 \\
RML & 0.21 & 0.33 \\
RLL & 0.01 & 0.90 \\
LUL & 0.03 & 0.80 \\
LLL & -0.10 & 0.14 \\
Multiple lobes & 0.08 & 0.41 \\
Extrapulmonary & 0.82 & 0.01 \\
Maternal age at term & 0.00 & 0.61 \\
Gravidity & 0.01 & 0.52 \\
Parity & -0.02 & 0.57 \\
\hline
\end{tabular}

Significant $p$ values $(p<0.05)$ have been underlined. n.a., not available; CPAM, congenital pulmonary airway malformation; BPS, bronchopulmonary sequestration; CLO, congenital lobar overinflation; bronchogenic cyst; RUL, right upper lobe; RML, right middle lobe; RLL, right lower lobe; LUL, left upper lobe; LLL, left lower lobe.

\section{Discussion}

We found that about $14 \%$ of the 196 CLA patients born and treated at our hospital over a 20 -year period had an associated anomaly or anomalies, which is a slightly higher proportion than the reported average of $11.7 \%$ in European countries [12]. The 3 patients with a lung agenesis all had associated anomalies, with a prevalence of $29 \%$ in patients with $\mathrm{BC}$ and a lower prevalence in patients with other CLAs. Congenital heart defects and gastrointestinal defects were the most common associated anomalies.

One of the patients in our cohort had both CPAM and BPS in one lung each. In contrast to our cohort, CLAs are most commonly associated with another type of CLA [1, 12]. In most cases, CPAM and BPS are reported in conjunction with each other, whereby the cystic CPAM lesion is accompanied by a more solid BPS component with systemic arterial blood supply. However, these lesions have recently been considered to comprise a single entity, the hybrid BPS [13]. Another known association is seen between bronchial atresia and both BPS and CLO, which occur in conjunction with other CLAs as well. Bronchial atre- sia is an abrupt interruption of the bronchus with a distal mucus plug, and it may be regarded as just a feature of some CLAs rather than as a true congenital abnormality [14].

In 2 cases, a different CLA was present in each lung, which supports the hypothesis that in all CLAs a common pathway is affected [15]. The exact cause of CLAs is still unknown, although it is hypothesized that there is an injury or arrest in normal lung development, the timing of which determines the type of CLA [7]. To date, no specific genetic factors have been identified, and only an estimated $1.6 \%$ of patients with a prenatally detected CLA have chromosomal anomalies [16]. Accordingly, 3 (1.5\%) patients in our entire cohort had a chromosomal anomaly, in each case an X-linked gene mutation. A CPAM patient had mosaic-type Klinefelter syndrome, an association which has only been reported twice before [17]. A CLO patient had an FLNA gene mutation, which is a known association [18]. And lastly, a lung agenesis patient had trisomy $\mathrm{X}$, which has not been reported previously. Some genetic conditions have indistinct clinical features, but there can be a great impact on the child's medical management and quality of life if the condition goes undetected [19]. Genetic counselling provides parents with information on the nature and natural history of a disorder, which may be useful for making informed decisions. We therefore recommend counselling by a clinical geneticist to assess the presence of genetic and syndromal abnormalities.

The diagnosis of $\mathrm{BC}$, lung agenesis, multiple CLA, and an extrapulmonary location significantly increased the odds of having an associated anomaly. Although not considered mid-line defects, lung agenesis and $\mathrm{BC}$ occur at the mid-line and presumably develop early in gestation [7, 15]. Similar to mid-line defects, the co-occurrence of defects in other organ systems may be more common as well [20]. In accordance with the literature, we found that $9 \%$ of our BPS patients had an associated anomaly, most often a gastrointestinal defect [21]. This supports suggestions that, like BC, extralobar BPS stems from a budding defect in which aberrantly located pulmonary mesenchyme develops [15]. In our cohort, the odds for associated anomalies was not significantly increased in patients with a BPS, most likely because we clustered intralobar, extralobar, and hybrid BPS, and only 1 patient in the group with associated anomalies had an extralobar BPS. Ridge regression coefficients are only an approximation of the influence of the parameters, therefore further research is needed to assess the exact influence. We recommend that clinicians remain vigilant for associated anomalies in patients with a BC, lung agenesis, or extrapulmonary CLA. 
In our cohort, associated congenital heart defects were most common. Previous studies reported that most CLAs remain asymptomatic, while other major congenital anomalies are regularly detected at a thorough newborn examination $[9,22]$. Thorough and structured newborn examinations may reveal congenital cardiac anomalies in the majority of cases [22]. Recently, pulse oximetry has been recommended as a non-invasive, cost-effective option for the early detection of critical congenital heart defects [22]. Cardiac ultrasound is the gold standard for non-invasive imaging of congenital cardiac anomalies, but it should only be performed when indicated, as most major congenital cardiac anomalies are diagnosed at a prenatal ultrasound or the newborn examination.

Signs of associated anomalies on chest and abdominal radiographs were seen in $9(32 \%)$ of the 28 patients. The routine use of chest radiographs in the postnatal management of CLA is under debate because the findings have limited impact on clinical management [23]. However, chest and abdominal radiographs may help distinguish between the subtypes of parenchymal and vascular pulmonary abnormalities, and they show signs of extrapulmonary abnormalities [24]. This can help to guide the choice of appropriate additional imaging. For example, a normal bowel gas pattern makes oesophageal or intestinal atresia less likely, and an intact and smooth hemi-diaphragm minimizes the probability of a congenital diaphragmatic hernia or eventration [25]. Hyperlucent lesions are more suggestive of BPS or BC, rather than CPAM, while an expanded hypolucent lesion may suggest CLO [24]. Skeletal anomalies of the vertebrae and ribs are often seen in patients with a lung agenesis and may also be found in association with a spinal neurenteric cyst [26]. In most of the patients in our cohort, associated anomalies were either detected prenatally (46\%) or at the newborn examination (39\%). Prenatal detection rates for major congenital anomalies are improving, especially when complemented by a thorough newborn examination [22]. For suspected postnatal cases, an antero-posterior and lateral chest and abdominal radiograph may be useful to assess signs of major associated anomalies.

A limitation of our study is that asymptomatic abnormalities may have been missed in the children born before 2007, the year when structured prenatal ultrasound screening was introduced in The Netherlands. While our structured follow-up programme is a valuable contribution, the retrospective data acquisition is another limitation of our study. Clinicians may have missed asymptomatic associated anomalies as additional investigation is not routine. Nevertheless, our cohort is a representative sample of the general population, because tertiary care is centralized in The Netherlands and our referral tertiary care centre serves a large, multi-cultural population. It was, however, a single-centre study, so the incidence found may differ for other populations.

In conclusion, associated anomalies were found in $14 \%$ of all patients with a CLA, especially those with lung agenesis and $\mathrm{BC}$. We suggest that during the newborn examination, special attention should be given to the cardiovascular and gastrointestinal tracts. An antero-posterior and lateral chest and abdominal radiograph may be useful to assess signs of major associated anomalies, regardless of the CLA type or clinical course, as the definitive CLA diagnosis is determined after cross-sectional imaging. Genetic counselling by a clinical geneticist should be offered to the parents of all patients. As lung agenesis and BC carry a higher risk for associated anomalies, especially cardiac anomalies, cardiac ultrasound is recommended in these cases to rule out a potentially fatal major cardiac anomaly.

\section{Acknowledgement}

We thank Ko Hagoort for critically reviewing the manuscript and providing editorial advice.

\section{Statement of Ethics}

The Erasmus MC Medical Ethics Review Board waived approval because data obtained during routine care were retrospectively analyzed (MEC-2018-1086).

\section{Conflict of Interest Statement}

The authors have no conflicts of interest to declare.

\section{Funding Sources}

This research did not receive any specific grant from funding agencies in the public, commercial, or not-for-profit sectors.

\section{Author Contributions}

S.M.H., R.R.Z., and J.M.S.: study conception and design. S.M.H. and R.R.Z.: retrospective chart review and data collection. S.M.H., R.R.Z., J.M.S., S.C.M.C.-O., R.M.H.W., and I.K.M.R.: data analysis and interpretation. All authors contributed to drafting and revising the manuscript for important intellectual content and approved the final version. 


\section{References}

1 Calzolari E, Barisic I, Loane M, Morris J, Wellesley D, Dolk H, et al. Epidemiology of multiple congenital anomalies in Europe: a EUROCAT population-based registry study. Birth Defects Res A Clin Mol Teratol. 2014 Apr;100(4):270-6.

2 Stocker LJ, Wellesley DG, Stanton MP, Parasuraman R, Howe DT. The increasing incidence of foetal echogenic congenital lung malformations: an observational study. Prenat Diagn. 2015 Feb;35(2):148-53.

3 Stanton $\mathrm{M}$. The argument for a non-operative approach to asymptomatic lung lesions. Semin Pediatr Surg. 2015 Aug;24(4):183-6.

4 Hermelijn SM, Elders BB, Ciet P, Wijnen RM, Tiddens HA, Schnater JM. A clinical guideline for structured assessment of CT-imaging in congenital lung abnormalities. Paediatr Respir Rev. 2020. DOI: 10.1016/j.prrv. 2019.12.004.

5 Singh R, Davenport M. The argument for operative approach to asymptomatic lung lesions. Semin Pediatr Surg. 2015 Aug;24(4): 187-95.

6 Gupta K, Das A, Menon P, Kakkar N, Rao KL, Joshi K. Revisiting the histopathologic spectrum of congenital pulmonary developmental disorders. Fetal Pediatr Pathol. 2012 Apr; 31(2):74-86.

7 Stocker JT. Congenital pulmonary airway malformation - a new name for and an expanded classification of congenital cystic adenomatoid malformation of the lung. Histopathology. 2002;(41):424-31.

8 Barriere G, Fici P, Gallerani G, Fabbri F, Rigaud M. Epithelial Mesenchymal Transition: a double-edged sword. Clin Transl Med. 2015 Apr;4(1): 14

9 WHO UNPF. UNICEF. Pregnancy, childbirth, postpartum and newborn care: A guide for essential practice. 3rd ed. Practice; 2015.
10 EUROCAT. EUROCAT Guide 1.4: Instruction for the registration of congenital anomalies. EUROCAT Central Registry. University of Ulster; 2013.

11 Golub GH, Heath M, Wahba G. Generalized Cross-Validation as a Method for Choosing a Good Ridge Parameter. Technometrics. 1979; 21(2):215-23

12 Moorthie S, Blencowe H, Darlison MW, Lawn J, Morris JK, Modell B, et al.; Congenital Disorders Expert Group. Estimating the birth prevalence and pregnancy outcomes of congenital malformations worldwide. J Community Genet. 2018 Oct;9(4):387-96.

13 Fowler DJ, Gould SJ. The pathology of congenital lung lesions. Semin Pediatr Surg. 2015 Aug;24(4):176-82.

14 Kunisaki SM, Fauza DO, Nemes LP, Barnewolt CE, Estroff JA, Kozakewich HP, et al. Bronchial atresia: the hidden pathology within a spectrum of prenatally diagnosed lung masses. J Pediatr Surg. 2006 Jan;41(1):61-5.

15 Langston C. New concepts in the pathology of congenital lung malformations. Semin Pediatr Surg. 2003 Feb;12(1):17-37.

16 Pressey TL, Wilson RD, Kasperski S, Bebbington MW, Adzick NS. Prenatal diagnosis of partial trisomy $1 \mathrm{q}$ and monosomy $\mathrm{X}$ in a fetus with a congenital lung lesion and hydrops fetalis. Am J Med Genet A. 2007 May; 143A(10):1104-7.

17 Holubyeva A, Bracero LA, Feuerstein JL, Bush S. Congenital pulmonary airway malformation associated with mosaic Klinefelter syndrome. J Clin Ultrasound. 2020 Feb;48(2): $121-4$.
18 Pelizzo G, Collura M, Puglisi A, Pappalardo MP, Agolini E, Novelli A, et al. Congenital emphysematous lung disease associated with a novel Filamin A mutation. Case report and literature review. BMC Pediatr. 2019 Mar; 19(1):86.

19 Ciarleglio LJ, Bennett RL, Williamson J, Mandell JB, Marks JH. Genetic counseling throughout the life cycle. J Clin Invest. 2003 Nov;112(9):1280-6.

20 Oyen N, Boyd HA, Poulsen G, Wohlfahrt J, Melbye M. Familial recurrence of midline birth defects-a nationwide danish cohort study. Am J Epidemiol. 2009 Jul;170(1):4652.

21 Wilson RD, Hedrick HL, Liechty KW, Flake AW, Johnson MP, Bebbington M, et al. Cystic adenomatoid malformation of the lung: review of genetics, prenatal diagnosis, and in utero treatment. Am J Med Genet A. 2006 Jan; 140(2):151-5.

22 Green $\mathrm{K}$, Oddie $\mathrm{S}$. The value of the postnatal examination in improving child health. Arch Dis Child Fetal Neonatal Ed. 2008 Sep; 93(5):F389-93.

23 Greig CJ, Keiser AM, Cleary MA, Stitelman $\mathrm{DH}$, Christison-Lagay ER, Ozgediz DE, et al. Routine postnatal chest $\mathrm{x}$-ray and intensive care admission are unnecessary for a majority of infants with congenital lung malformations. J Pediatr Surg. 2019 Apr;54(4):670-4.

24 Lee EY, Dorkin H, Vargas SO. Congenital pulmonary malformations in pediatric patients: review and update on etiology, classification, and imaging findings. Radiol Clin North Am. 2011 Sep;49(5):921-48.

25 Prasad GR, Aziz A. Abdominal Plain Radiograph in Neonatal Intestinal Obstruction. J Neonatal Surg. 2017 Jan;6(1):6.

26 Vargas D, Suby-Long T, Restrepo CS. Cystic Lesions of the Mediastinum. Semin Ultrasound CT MR. 2016 Jun;37(3):212-22. 